Relato de Caso

CASE REPORT

\title{
Uso da Oxigenoterapia Hiperbárica em Pacientes de um Serviço de Reumatologia Pediátrica ${ }^{*}$
}

\section{Hyperbaric Oxysen Therapy in Patients of a Pediatric Rheumatolosy Service}

\author{
Juliana Figueira M. R. Rossi ${ }^{(1)}$, Polyanna Maria Ferreira Soares ${ }^{(2)}$, Bernadete Lourdes Liphaus ${ }^{(3)}$, \\ Mariza D’Agostino Dias ${ }^{(4)}$, Clovis Artur Almeida Silva ${ }^{(5)}$
}

\section{RESUMO}

A oxigenoterapia hiperbárica consiste na respiração de oxigênio puro sob pressão maior que uma atmosfera e vem sendo empregada no tratamento adjuvante de lesões ulceradas de várias etiologias. Foram analisados retrospectivamente os prontuários de seis pacientes atendidos no Ambulatório de Reumatologia Pediátrica do Instituto da Criança do Hospital das Clínicas da FMUSP entre 1996 e 2002, submetidos à oxigenoterapia hiperbárica. Esse procedimento foi indicado pela presença de osteomielite crônica e lesões ulceradas (por vasculite ou infecção) que não responderam ao tratamento habitual. Dois pacientes apresentaram poliarterite nodosa cutânea, dois osteomielite crônica multifocal recorrente, um esclerodermia cutânea difusa e um pioderma gangrenoso. Cinco pacientes eram do sexo feminino (idade variou de 6 a 13,2 anos). As sessões de oxigenoterapia hiperbárica foram realizadas sob pressões que variaram de 2,4 a 2,8 atmosferas absolutas, com duração de duas horas. O número mínimo de sessões foi 18 e o máximo 80. Em cinco pacientes houve completa cicatrização das lesões. A paciente com esclerodermia cutânea abandonou o tratamento após a décima oitava sessão, pois retornou a sua cidade natal, com melhora parcial das lesões cutâneas. O principal evento adverso durante as sessões foi otalgia, após as primeiras sessões, que desapareceu com diminuição da pressão dentro da câmara e da duração da sessão. Não foram observados perfuração timpânica ou outros eventos adversos. A oxigenoterapia hiperbárica foi eficaz e bem tolerada nos pacientes com doenças reumatológicas e lesões vasculíticas ulceradas ou infectadas e osteomielite crônica.

Palavras-chave: oxigenoterapia hiperbárica, esclerodermia, vasculite, osteomielite, crianças e adolescentes.

\begin{abstract}
The hyperbaric oxygen therapy consists in the breathing of pure oxygen under pressures higher than one atmosphere and has been used in the treatment of ulcerated lesions of various etiologies. Six patients followed at the Rheumathology Unit, Instituto da Criança, Hospital das Clínicas, Faculty of Medicine, São Paulo University, between 1996 and 2002, were submitted to hyperbaric oxygen therapy. This therapy was indicated by the presence of chronic osteomyelitis and tissue ulcer (vasculitis or infection) not responsive to the usual treatment. Two patients presented cutaneous polyarteritis, two presented chronic recurrent multifocal osteomyelitis, one presented diffuse cutaneous sclerodema and one presented pyoderma gangrenosum. Five patients were girls (age range from 6 to 13.2 years-old). The sessions of hyperbaric oxygen therapy were performed under pressures that ranged from 2.4 to 2.8 absolute atmospheres and their duration were two hours. The lowest number of sessions was 18 and the highest was 80 . Five patients presented complete resolution of the injuries. The patient with cutaneous sclerodema suspended the treatment after the 18th session, because she went back to her birthplace with partial improvement of the cutaneous injuries. The main adverse event during the sessions was ear pain after the first sessions, which disappeared with reduction of the pressure inside the chamber and the duration of the session. Spandrel perforation or other adverse events were not observed. The hyperbaric oxygen therapy was efficient and well tolerated by patients with rheumatic diseases and ulcerated injuries by vasculitis, infected injuries or chronic osteomyelitis.
\end{abstract}

Keywords: hyperbaric oxygen therapy, scleroderma, vasculitis, osteomyelitis, children, adolescents.

\footnotetext{
* Trabalho realizado na Unidade de Reumatologia Pediátrica do Departamento de Pediatria e Serviço de Oxigenoterapia Hiperbárica do Hospital das Clínicas da Faculdade de Medicina da Universidade de São Paulo (FMUSP). Recebido em 18/08/2004. Aprovado, após revisão, em 27/01/2005

1. Médica complementanda da Unidade de Reumatologia Pediátrica do Departamento de Pediatria da FMUSP.

2. Médica pós-graduanda da disciplina de Reumatologia da FMUSP.

3. Mestre em Medicina pela FMUSP. Médica assistente da Unidade de Reumatologia Pediátrica do Departamento de Pediatria da FMUSP.

4. Médica-supervisora da Unidade de Terapia Intensiva do Trauma do Hospital das Clínicas da FMUSP. Médica Responsável pelo Serviço de Oxigenoterapia Hiperbárica do Hospital das Clínicas da FMUSP.

5. Doutor em Medicina pela FMUSP. Médico chefe da Unidade de Reumatologia Pediátrica do Departamento de Pediatria da FMUSP.

Endereço para correspondência: Dr. Clovis Artur Almeida da Silva. R. Senador César Lacerda Vergueiro, 494/82, V. Madalena, CEP 05435-010, São Paulo, SP, Brasil. Tels. (1 1) 3069-8675/3069-8503; e-mail:clovisaas@icr.hcnet.usp.br
} 


\section{INTRODUÇÃO}

A oxigenoterapia hiperbárica consiste na exposição do paciente à respiração de oxigênio puro a 100\%, em temperatura ambiente, sob pressão maior que uma atmosfera absoluta ${ }^{(1)}$.

Essa terapêutica é fundamentada em duas leis físicas e em alguns processos bioquímicos que explicam seu mecanismo de ação: a lei de Henry, segundo a qual a "quantidade de um gás que se dissolve em um líquido (neste caso, o oxigênio no plasma) é tanto maior quanto maior for a pressão exercida por este gás sobre esse líquido", e a lei de Boyle-Mariotti, segundo a qual "o espaço ocupado por um volume de gás será cada vez menor quanto maior for a pressão ambiente", ou seja, em um ambiente pressurizado o gás sofre contração, expandindo-se com a diminuição da pressão ${ }^{(2)}$.

O mecanismo de ação da oxigenoterapia hiperbárica é o resultado de uma combinação de fatores. Leucócitos em hipóxia apresentam dificuldade na fagocitose de bactérias e esta função se restaura com a elevação da tensão de oxigênio. Além disso, a síntese de colágeno pelos fibroblastos aumenta com a maior disponibilidade de oxigênio ${ }^{(3)}$. A oxigenação tecidual obtida pela oxigenoterapia hiperbárica restaura a angiogênese capilar, aumentando a proliferação tecidual e a formação de tecido de granulação ${ }^{(4,5)}$. Em pacientes tratados com oxigenoterapia hiperbárica ocorre aumento na pressão parcial de oxigênio no sangue arterial com concomitante elevação do gradiente de oxigênio entre os capilares e os tecidos, aumentando a oxigenação celular e quebrando o ciclo vicioso da isquemia ${ }^{(6)}$. Existe vasoconstrição e conseqüente redução de edemas e de pressões compartimentais durante o tratamento com oxigenoterapia hiperbárica ${ }^{(7)}$.

Recentes evidências mostram que a oxigenoterapia hiperbárica inibe a ação de certas citocinas, como a interleucina 1. Esta citocina também atua como imunomodulador, por elevar os níveis séricos de linfócitos T CD8 e diminuir os de linfócitos T CD4, sem alterar os níveis dos linfócitos T CD3, linfócitos B e células natural killer, justificando o seu uso em algumas doenças reumáticas ${ }^{(8)}$. Estudos com esse método terapêutico na reumatologia pediátrica têm sido raramente publicados.

O objetivo deste trabalho foi descrever o uso da oxigenoterapia hiperbárica como tratamento adjuvante nos pacientes com doenças reumatológicas com lesões cutâneas, musculares ou ósseas por atividade de doença, associadas ou não a infecção. Os pacientes foram seguidos na Unidade de Reumatologia Pediátrica do Instituto da Criança e realizaram oxigenoterapia hiperbárica no Serviço de Oxigenoterapia Hiperbárica da Cirurgia do Trauma do Hospital das Clínicas da FMUSP.

\section{RELATO DE CASOS}

Seis crianças e adolescentes com doenças reumatológicas, que apresentaram lesões cutâneas, lesões de partes moles ou ósseas por atividade de doença, associadas ou não a infecção, e que não responderam ao tratamento habitual foram submetidas à oxigenoterapia hiperbárica. As principais características demográficas e clínicas dos pacientes, bem como dados da oxigenoterapia hiperbárica, estão listados na Tabela 1.

TABELA 1

CARACTERÍSTICAS DEMOGRÁFICAS E CLÍNICAS DOS PACIENTES E USO DA OXIGENOTERAPIA HIPERBÁRICA DE SEIS PACIENTES COM DOENÇAS REUMATOLÓGICAS PEDIÁTRICAS

\begin{tabular}{|c|c|c|c|c|c|c|}
\hline Características & $\begin{array}{c}\text { Paciente } \\
1\end{array}$ & $\begin{array}{c}\text { Paciente } \\
2\end{array}$ & $\begin{array}{c}\text { Paciente } \\
3\end{array}$ & $\begin{array}{c}\text { Paciente } \\
4\end{array}$ & $\begin{array}{l}\text { Paciente } \\
5\end{array}$ & $\begin{array}{c}\text { Paciente } \\
6\end{array}$ \\
\hline Idade (anos) & 11 & 13 & 6 & 9 & 12 & 6 \\
\hline Sexo & $\mathrm{F}$ & $\mathrm{F}$ & $\mathrm{F}$ & $\mathrm{F}$ & $\mathrm{F}$ & M \\
\hline Diagnóstico & OCMR & OCMR & PANC & PANc & ECD & PG \\
\hline VHS (mm 1a hora) & 35 & 55 & 70 & 52 & 62 & 35 \\
\hline Tempo de tratamento (meses) & 6 & 7 & 2 & 3 & 1 & 3 \\
\hline Indicação da $\mathrm{OH}$ & $A, I$ & $A, I$ & A, I & A, I & $A, I$ & A \\
\hline Número total de sessões da $\mathrm{OH}$ & 58 & 80 & 19 & 32 & 18 & 39 \\
\hline Evento adverso associado à $\mathrm{OH}$ & Otalgia & Otalgia & Dor em quirodáctilos & Cianose e frio & - & Otalgia \\
\hline Tratamento & AINH, ATB & AINH, ATB, MTX & $\mathrm{Pd}, \mathrm{C}, \mathrm{GGEV}$ & $\mathrm{Pd}, \mathrm{C}$ & Pd, MTX, DPA & $\mathrm{Pd}, \mathrm{C}, \mathrm{Cp}$ \\
\hline
\end{tabular}

+ = presente; $\mathrm{A}=$ atividade; $\mathrm{AINH}=$ antiinflamatório não hormonal; $\mathrm{ATB}=$ antibiótico; $\mathrm{C}=$ ciclofosfamida; $\mathrm{Cp}=$ ciclosporina; $\mathrm{DPA}=\mathrm{D}$-penicilamina; $\mathrm{ECD}=$ esclerodermia cutânea difusa; $\mathrm{F}=$ feminino; $\mathrm{GGEV}=$ gamaglobulina endovenosa; $\mathrm{I}=$ infecção; $\mathrm{M}=$ masculino; $\mathrm{MTX}=$ metotrexato; $0 \mathrm{CR}=$ osteomielite crônica multifocal recorrente; $0 \mathrm{H}=0$ oxigenoterapia hiperbárica; $\mathrm{PANc}=$ poliarterite nodosa cutânea; $\mathrm{Pd}=$ prednisona; $\mathrm{PG}=$ pioderma gangrenoso; $\mathrm{VHS}=$ velocidade de hemossedimentação 
Os pacientes receberam o tratamento para suas respectivas doenças de base (antiinflamatórios não hormonais, corticosteróides e/ou imunossupressores), simultaneamente à oxigenoterapia hiperbárica. Quando havia infecção secundária, foram instituídos antibioticoterapia e cuidados locais.

A oxigenoterapia hiperbárica foi realizada com oxigênio a $100 \%$ sob pressões que variaram de 2,4 a 2,8 atmosferas, com duração de duas horas. O número e o intervalo das sessões variaram entre os pacientes. O número mínimo de sessões foi 18 e o máximo 80 (média de 41 sessões), com intervalos que variaram de dois a sete dias entre as sessões.

Com relação às indicações da oxigenoterapia hiperbárica: duas pacientes apresentavam poliarterite nodosa cutânea em atividade (uma com úlceras vasculíticas no membro superior direito e falanges distais da mão direita e outra com lesão ulcerada e necrótica no pé direito); duas tiveram osteomielite crônica multifocal recorrente, exclusivamente, de mandíbula refratária a antibioticoterapia; uma apresentava esclerodermia cutânea difusa com várias úlceras infectadas na perna direita (Figuras 1 e 2) e um paciente apresentou lesões difusas de pioderma gangrenoso idiopático.

Dois pacientes utilizaram a oxigenoterapia hiperbárica em regime ambulatorial (pacientes 2 e 4); três pacientes estavam internados no início das sessões, com posterior realização ambulatorial (pacientes 1, 3 e 6) e apenas uma paciente permaneceu internada em todas as sessões por motivo social (paciente 5).

Em cinco pacientes houve completa cicatrização das lesões. A paciente com esclerodermia cutânea abandonou o tratamento após a décima oitava sessão, pois retornou para a sua cidade natal, com melhora parcial das úlceras. A melhora das lesões ocorreu em média após a vigésima primeira sessão de oxigenoterapia hiperbárica. Nos pacientes com osteomielite crônica multifocal recorrente houve um início de melhora da dor e edema em região mandibular após 15 e 38 sessões, respectivamente (pacientes 2 e 1 ).

O principal evento adverso durante as sessões de oxigenoterapia hiperbárica foi otalgia, referida por três pacientes especialmente no início do tratamento. A otalgia desapareceu com a diminuição da pressão dentro da câmara e da duração das primeiras sessões. Não foi observada perfuração de membrana timpânica. Dor em quirodáctilos foi relatado pelo paciente 3 que apresentava lesões ulceradas no membro superior direito. Cianose e frio foram referidos pelo paciente 4 que apresentava lesão ulcerada no pé direito. Não foram observados outros eventos adversos.

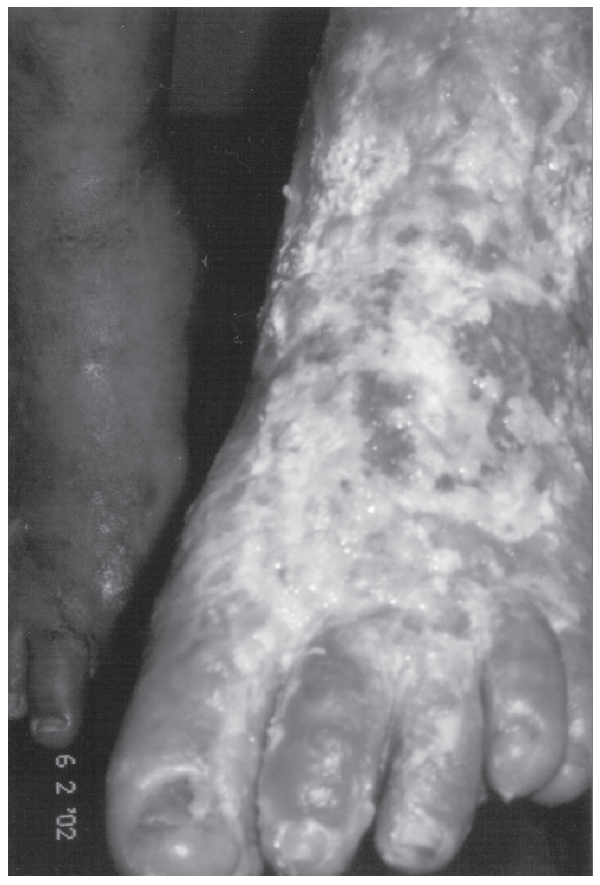

Figura 1 - Esclerodermia cutânea difusa com lesões ulceradas infectadas em membro inferior esquerdo (Paciente 5)

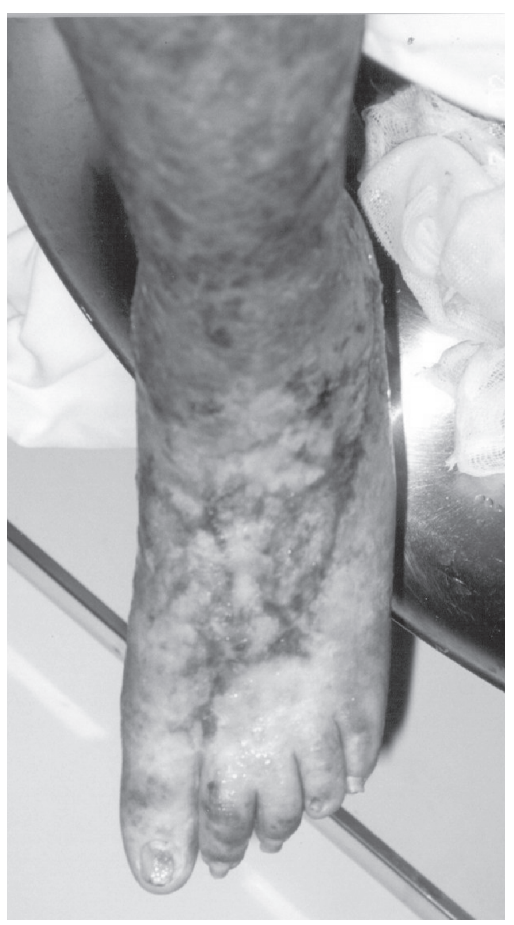

Figura 2 - Esclerodermia cutânea difusa que apresentou melhora das lesões após a $18^{a}$ sessão de oxigenoterapia hiperbárica (Paciente 5) 


\section{DISCUSSÃO}

A oxigenoterapia hiperbárica é um tratamento seguro na faixa etária pediátrica quando usada de forma criteriosa, com técnicas adequadas e por pessoas treinadas.

Esta terapêutica tem sido indicada em infecções necrosantes de tecidos moles, isquemias periféricas agudas, gangrena gasosa, osteomielite aguda de risco ou crônica refratária, intoxicação por monóxido de carbono e cianeto, enxertos e retalhos comprometidos e de alto risco, micoses refratárias, necrose por radiação e em embolia gasosa ${ }^{(7)}$. As contra-indicações absolutas de oxigenoterapia hiperbárica incluem pneumotórax não drenado e uso de terapia com cis-platinum ou dissulfiram ${ }^{(1)}$.

É importante ressaltar que o oxigênio sob condições hiperbáricas age como uma medicação, que exerce efeitos específicos no corpo humano que não estão presentes a uma pressão normal. O oxigênio hiperbárico tem contraindicações, eventos adversos e interage também com outros medicamentos ${ }^{(8)}$.

O tempo das sessões de oxigenoterapia hiperbárica na literatura varia, em geral, de 30 minutos a duas horas e o número de sessões de 15 a $40^{(1,6,8)}$. Nos pacientes deste estudo, o tempo de duração das sessões foi de duas horas e o número de sessões variou de 18 a 80 . Os dois pacientes que necessitaram de maior número de sessões, para regressão progressiva da dor e da tumoração mandibular, apresentavam osteomielite crônica multifocal recorrente de mandíbula.

A osteomielite crônica multifocal recorrente é uma doença inflamatória óssea rara, com acometimento raro de mandíbula, com períodos de exacerbação e remissão e habitualmente sem agentes infecciosos isolados nas áreas afetadas. O tratamento é realizado com antiinflamatórios não hormonais, corticosteróides ou metotrexato. A oxigenação hiperbárica pode ser usada como terapêutica adjuvante nos casos refratários ${ }^{(9)}$, como foi utilizada em dois de nossos casos.

O oxigênio hiperbárico atua de forma sinérgica com os antibióticos, porque modifica o ambiente bioquímico tornando-o desfavorável à proliferação bacteriana, limitando a produção e interferindo na atividade de suas toxinas, além de ser diretamente bactericida para os germes anaeróbios ${ }^{(10)}$. Em cinco de nossos pacientes acreditamos ter ocorrido este tipo de sinergia, pois apresentavam lesões infectadas (dois osteomielite crônica e três lesões cutâneas e de partes moles) que não estavam respondendo à antibioticoterapia convencional.
Os mecanismos de ação da oxigenoterapia hiperbárica incluem proliferação do epitélio, formação de tecido de granulação, estimulação de fibroblastos, aumento da produção de colágeno, angiogênese capilar e alteração da dinâmica do tecido lesado, auxiliando a cicatrização tecidual ${ }^{(3,4)}$.

Davis et al. avaliaram quatro pacientes (idades entre 17 e 64 anos) com pioderma gangrenoso tratados com oxigenoterapia hiperbárica no pré-operatório de enxerto cutâneo e observaram que todos responderam com redução de infecção e aumento de neovascularização ${ }^{(11)}$. $\mathrm{O}$ nosso paciente com pioderma gangrenoso não necessitou de enxerto cutâneo, pois apresentou boa resposta ao tratamento com oxigenoterapia hiperbárica, com epitelização completa dessas áreas.

A isquemia tecidual afeta tecido nervoso, músculos e leito capilar. O edema decorrente da isquemia tecidual piora ainda mais a oxigenação tecidual. A oxigenoterapia hiperbárica torna-se uma importante indicação neste caso, no intuito de minimizar o dano tecidual, que é proporcional ao tempo de anóxia ${ }^{(5,6)}$. Provavelmente, um menor tempo de anóxia tecidual foi o responsável pela melhora observada nos dois pacientes com poliarterite nodosa cutânea deste estudo, que apresentavam lesões vasculíticas isquêmicas de extremidades.

Os eventos adversos da oxigenoterapia hiperbárica estão relacionados às alterações de volume e pressão e à toxicidade do oxigênio. O mais comum é o barotrauma de ouvido (com otalgia persistente) e em casos extremos com hemorragia e perfuração timpânica. Outras complicações raras são: toxicidade pulmonar (com tosse, irritação de vias aéreas superiores e diminuição da capacidade vital); aceleração do processo de catarata; toxicidade em sistema nervoso central (irritabilidade, diminuição de campo visual, náusea, tontura e convulsão generalizada) ${ }^{(2)}$. Neste estudo, o único evento adverso observado foi otalgia, que melhorou após redução da pressão e da duração das sessões de oxigenoterapia hiperbárica.

A prática de oxigenoterapia hiperbárica está regulamentada no Brasil pelo Conselho Federal de Medicina (CFM) desde $1995^{(12,13)}$. Os pontos mais importantes são os seguintes: a indicação de oxigenoterapia hiperbárica é de exclusiva competência médica; a aplicação de oxigenoterapia hiperbárica deve ser realizada por médico ou sob sua supervisão; outros tipos de tratamento, tais como uso de oxigênio em tendas, ou em respiradores, mesmo a $100 \%$, não são caracterizados como oxigenoterapia hiperbárica. Dentre as indicações de oxigenoterapia hiperbárica 
aceitas pelo CFM estão as vasculites agudas, lesões refratárias (úlceras de pele e úlceras por vasculites auto-imunes) e osteomielites.

O pequeno conhecimento desta modalidade terapêutica, a pouca experiência de sua utilização em crianças e a sua disponibilidade em poucos serviços são fatores limitantes de seu uso.

\section{REFERÊNCIAS}

1. Wallace DJ, Silverman S, Goldstein J, Hughes D: Occasional Review. Use of Hyperbaric oxygen in rheumatic diseases: case report and critical analysis. Lupus 4: 172-5, 1995.

2. Waisman D, Shupak A, Weisz G, Melamed Y: Hyperbaric Oxygen Therapy in the Pediatric Patient: The Experience of the Israel Naval Medical Institute. Pediatrics 102: 53, 1998.

3. Wasserteil V, Bruce S, Sessoms SL, Guntupalli KK: Pyoderma Gangrenosum treated with Hyperbaric Oxygen Therapy. Int J Dermatol 31: 594-6, 1992.

4. Weisz G, Ramon Y, Waisman D, Melamed Y: Treatment of Necrobiosis Lipoidica Diabeticorum by Hyperbaric Oxygen. Acta Derm Venereol 73: 447-8, 1993.

5. Heng MCY: Hyperbaric Oxygen Therapy for a foot ulcer in a patient with Polyarteritis Nodosa. Aust J Derm 24: 105-8, 1983.

6. Monies-Chass I, Herer D, Alon U, et al: Hyperbaric Oxygen in acute ischaemia due to allergic vasculitis. Anaesthesia 31: 1221-4, 1976.
Acreditamos que o uso de oxigenoterapia hiperbárica auxiliou na redução da morbidade, da letalidade, do tempo de internação, do consumo de antibióticos e conseqüentemente dos custos totais do tratamento de nossos pacientes. Portanto, a oxigenoterapia hiperbárica mostrou-se um recurso terapêutico adjuvante bem tolerado e eficaz no tratamento de lesões ulceradas vasculíticas ou infectadas e osteomielite crônica.

7. Jon T, Kenneth R, Adams M, William R: Efeitos Fisiológicos do Oxigênio Hiperbárico. J Trauma 15: 1-3, 1993.

8. Sallusti R, Ferraù $\mathrm{S}$, Valdes $\mathrm{AL}$, et al: Education and training in Hyperbaric medicine. The Icelandic experience. Minerva Anestesiol 67: 713-25, 2001.

9. Paim LB, Liphaus BL, Rocha AD, Castellanos AL, Silva CA: Osteomielite crônica multifocal recorrente (CRMO) da mandíbula: relato de três casos. J Pediatr (Rio J) 79: 467-70, 2003.

10. Kronish JW, McLeish MW: Hyperbaric Oxygen Therapy in Infections: In Kronish JW: Textbook of Hyperbaric Medicine. Lewiston NY: Hogrefe \& Huber Publishers, 1996. p. 178-99.

11. Davis JC, Landeen JM, Levine RA: Pyoderma Gangrenosum: Skin Grafting after Preparation with Hyperbaric Oxygen. Plast Reconstr Surg 79: 200-6, 1987.

12. Resolução do Conselho Federal de Medicina 1457/95 sobre oxigenoterapia hiperbárica: DOU out. 19 seção 1:16585, 1995.

13. Resolução do Conselho Federal de Medicina 1457/95 sobre oxigenoterapia hiperbárica: DOU nov. 30 seção 1: 19829, 1995. 